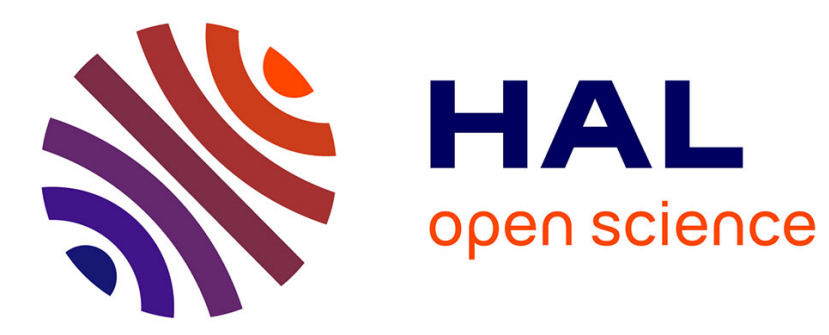

\title{
CARACTERISATION D'UN LASER Nd: YAG CONTINU A MODES COUPLES
}

\author{
B. Geoffroy, A. Haessler, F. Heisel, T. Lépine, J. Miehé
}

\section{To cite this version:}

B. Geoffroy, A. Haessler, F. Heisel, T. Lépine, J. Miehé. CARACTERISATION D'UN LASER Nd: YAG CONTINU A MODES COUPLES. Journal de Physique Colloques, 1987, 48 (C7), pp.C7-409C7-411. 10.1051/jphyscol:1987798 . jpa-00227103

\section{HAL Id: jpa-00227103 https://hal.science/jpa-00227103}

Submitted on 1 Jan 1987

HAL is a multi-disciplinary open access archive for the deposit and dissemination of scientific research documents, whether they are published or not. The documents may come from teaching and research institutions in France or abroad, or from public or private research centers.
L'archive ouverte pluridisciplinaire HAL, est destinée au dépôt et à la diffusion de documents scientifiques de niveau recherche, publiés ou non, émanant des établissements d'enseignement et de recherche français ou étrangers, des laboratoires publics ou privés. 


\title{
CARACTERISATION D'UN LASER Nd:YAG CONTINU A MODES COUPLES
}

\author{
B. GEOFFroy, A. HAESSLER, F. HEISEL, T. LEPINE et J.A. MIEHE \\ CRN, Groupe d'Optique Appliquée et Groupe de photophysique \\ Moléculaire, BP 20,F-67037 Strasbourg Cedex, France
}

\begin{abstract}
The temporal characteristics of the frequency doubled pulses of a continuous wave mode locked Nd:YAG 1 aser have been investigated by means of a streak camera. Single shot streak measurements allow direct studies of the individual laser pulses. The stability in amplitude and shape of the pulses depends on the critical alignment of the mode locker and on the cavity length adjustment. Synchroscan measurements made conspicuous the influence of the spectral quality of the oscillator on the fluctuations of the temporal profil, of the intensity and also of the time interval between successive pulses.
\end{abstract}

L'optimalisation des performances d'un laser Nd:YAG a été entreprise d'une part en se basant sur les résultats mettant en évidence l'influence des différents constituants de la cavité [1], d'autre part en ajustant les paramètres dynamiques du laser pour obtenir une excellente stabilité de fonctionnement contrôlée à l'aide d'une caméra à balayage de fente utilisée en mode déclenché au coup par coup et au taux de répétition des impulsions du laser $(82 \mathrm{MHz})$.

Les divers histogrammes enregistrēs au moyen des deux dispositifs expérimentaux décrits précédemment [2] ont permis un traitement statistique des données pour caractériser les fluctuations -à court et à long termes- de la forme temporelle et de l'intensité lumineuse des impulsions ainsi que la dispersion des intervalles de temps entre événeménts successifs.

Sur la figure 1 est représenté un échantillonnage d'enregistrements au coup par coup ainsi que les variations correspondantes au cours du temps ( $\sim 5 \mathrm{mn})$ de la largeur à mi-hauteur totale et de l'amplitude des enregistrements.

Compte tenu des fluctuations statistiques dues au faible éclairement de la photocathode de la caméra à balayage de fente, on déduit que la stabilité en intensité et en durée des impulsions délivrëes par le laser est nettement meilleure que $20 \%$.

L'importance de la stabilité et de la pureté spectrale de l'oscillateur pilotant le coupleur de modes est illustrée sur la figure 2 reprësentant les histogrammes temporels relevés avec une caméra à balayage de fente fonctionnant en mode de déflexion sinusoīdale (82 $\mathrm{MHz}$ ), pour deux générateurs sinusoĩdaux $(41 \mathrm{MHz})$ de largeurs spectrales respectivement égales à 750 et à $380 \mathrm{~Hz}$ à $-40 \mathrm{~dB}$.

Les pics successifs des histogrammes résultent de l'emploi d'un Fabry-Përot servant au calibrage en temps et en intensité. La comparaison des résultats montre que les largeurs à mi-hauteur des pics ne sont pas affectées par le bruit visible sur le relevé de l'analyse spectrale de l'oscillateur. Par contre, il est nettement apparent que les rapports pic/vallée sont différents : 


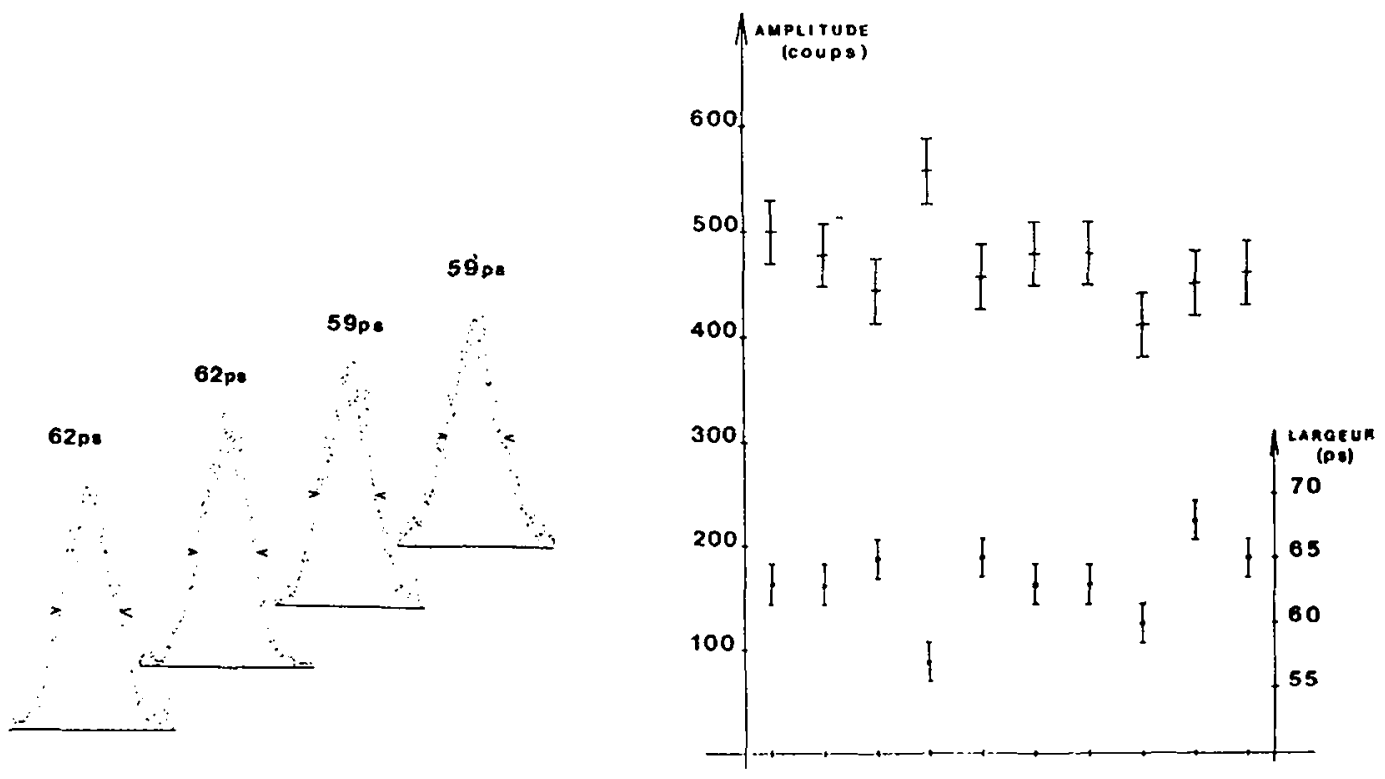

Fig.1 : Histogrammes d'impulsions lumineuses uniques $(\lambda=532 \mathrm{~nm})$. Courbes de variations des durēes et des intensités crêtes de ces impulsions au cours du temps
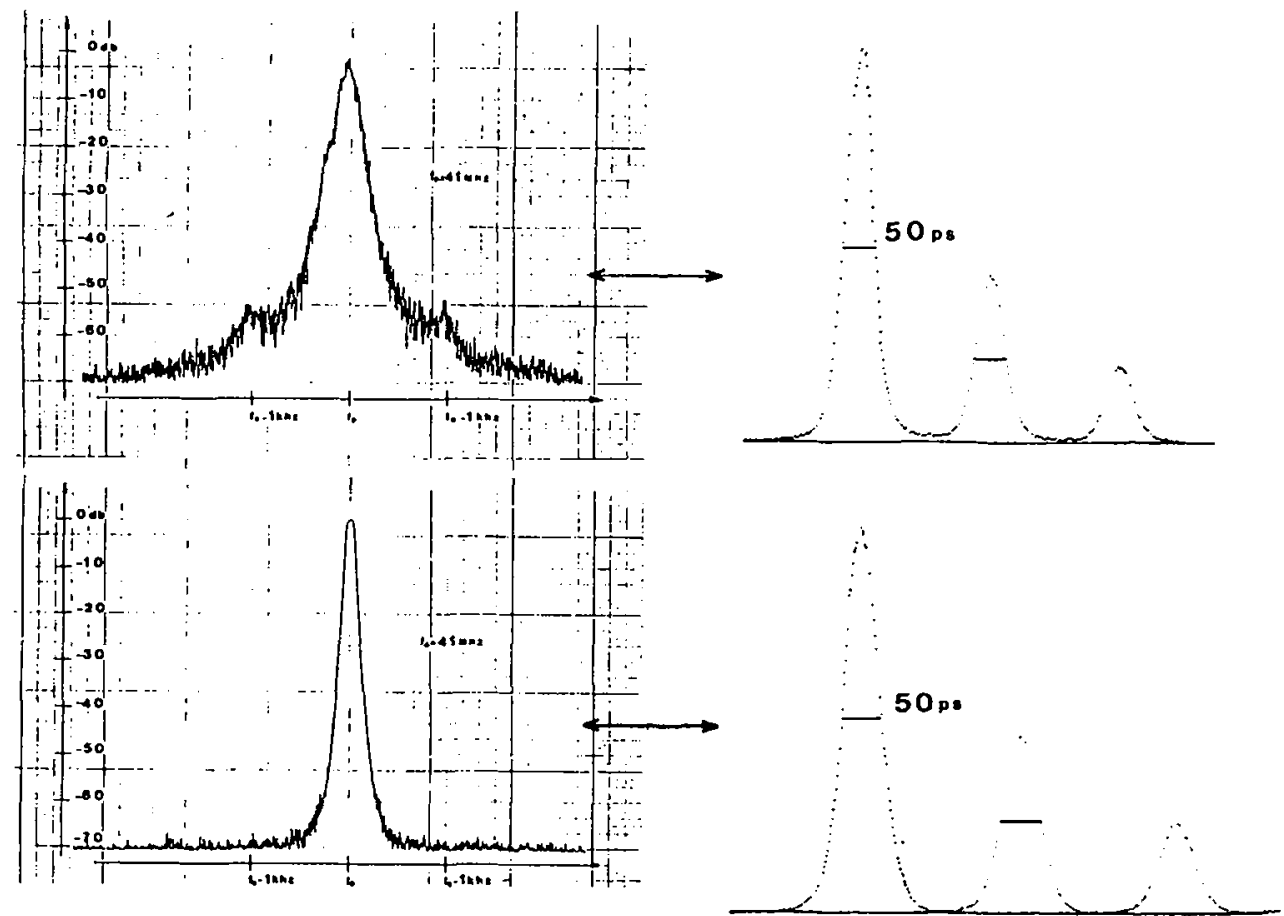

Fig.2 : Relevés de l'analyse spectrale de deux oscitlateurs sinusoĩdaux et histogrammes des impulsions $(\lambda=532 \mathrm{~nm})$ obtenus à l'aide d'une caméra à balayage de fente fonctionnant en mode sinusoidal 
une analyse des impulsions uniques dans les mêmes conditions de fonctionnement du laser a mis en évidence des instabilités en durée et en intensité très chaotiques pour l'oscillateur à largeur spectrale élevée.

1. V. Magni, Applied Optics, 25, $n^{\circ} 1$ (1986) 107

2. P. Geist, F. Heisel, A. Martz et J.A. Miehé, Revue de Physique Appliquée $19(1984) 619$ 